\title{
The clinical and histopathological characteristics of Kelly punch punctoplasty
}

\author{
Ayelet Priel ${ }^{1,2} \cdot$ Mordechai Rosner $^{1,2} \cdot$ Guy Ben Simon ${ }^{1,2} \cdot$ Jonathan Weidenfeld $^{2,3} \cdot$ Alon Weissman $^{1,2} \cdot$ \\ Daphna Prat $\mathbb{D}^{1,2} \cdot$ Ofira Zloto ${ }^{1,2}$
}

Received: 2 July 2019 / Revised: 29 September 2019 / Accepted: 4 November 2019 / Published online: 17 February 2020

(c) The Author(s), under exclusive licence to The Royal College of Ophthalmologists 2020

\begin{abstract}
Purpose To examine the clinical presentation, histopathological characteristics and surgical success of patients that underwent Kelly punch punctoplasty.

Methods A retrospective consecutive case series of all patients diagnosed with punctal stenosis and referred to Kelly punch punctoplasty and mini-monoka insertion between January 2017 and December 2017. The demographic, clinical and prognosis parameters were retrieved and analyzed. Moreover, a histopathological examination was performed on the tissue retrieved from the Kelly punch at the end of the procedure in order to evaluate the presence of muscle in the tissue, as well as chronic inflammation.

Results The series was composed of 44 eyes of 28 patients. All patients' complaints prior to surgery were epiphora: 18 patients had bilateral epiphora, 5 presented with right-eye epiphora and 5 with left-eye epiphora. The average Munk degree of epiphora before surgery was 3 and at least 3 months after surgery 1.04 (matched pairs, $p<0.0001$ ). Two patients underwent the second punctoplasty. In haematoxylin and eosin staining, the Riolan muscle was not visible in the mucosal layer in 40 specimens. In four specimens, the muscle was observed. A Masson's trichrome staining ensured that the Riolan muscle existed only in those four specimens.

Discussion Kelly punch punctoplasty is a simple and minimally invasive procedure for punctal dilation with high functional success rate. Moreover, in histopathological specimens, in all but four of the cases there was no muscle indicated. Intact Riolan muscle enables the punctum to stay in the right circular shape and position, and the lacrimal pump to maintain its pressure gradient.
\end{abstract}

\section{Introduction}

Punctal stenosis is a common reason for epiphora [1]. Many aetiologies have been associated with acquired punctal stenosis. These include involutional, infectious causes (such as Actinomyces or Chlamydia trachomatis), autoimmune and inflammatory conditions (such as dry eye, chronic blepharitis, ectropion and graft-versus-host disease).

Ayelet Priel

priel.ayelet@gmail.com

1 Goldschleger Eye Institute, Sheba Medical Center, Tel Hashomer, Israel

2 Sackler Faculty of Medicine, Tel Aviv University, Tel Aviv, Israel

3 Institute of Pathology, Sheba Medical Center, Tel Hashomer, Sackler Faculty of Medicine, Tel-Aviv University, Tel Aviv, Israel
Moreover, several medications have been associated with punctal stenosis, both topical and systemic. Common medications causing punctual stenosis are 5-FU, Taxols and topical glaucoma medications [2].

In 1851, Bowman was the first to describe the one-snip punctoplasty [3]. Since then, different types of snip punctoplasty have been developed including one- to fourdirection snip punctoplasty [4, 5]. However, restenosis due to the healing scar of the snip edges, and disruption of canaliculus anatomy leading to pumping disturbances, have been reported [2]. Therefore, it has been recommended to add adjuvant treatments, such as mitomycin $\mathrm{C}$ [6], punctal plug insertion and mini-monoka stents [7]. However, restenosis remained a main concern [8]. Therefore, the search for a promising solution for punctal stenosis continued.

In 1967, Hughs et al. reported their experience with the use of a punch to perform posterior ampullectomy [9]. Almost 25 years later, Edelstein and Reiss introduced a 
special instrument named the Reiss punctal punch [10]. This technique did not become popular. However, in 2011, Carrim et al. suggested to use Kelly Descemet's membrane punch for punctoplasty, and since then this procedure became more popular maybe due to availability of the Kelly punch in most ophthalmology-operating rooms [11].

The lacrimal puncta are the two entry points from the ocular surface to the lacrimal system. The lower lacrimal punctum is a small opening, $\sim 0.3 \mathrm{~mm}$, at the medial aspect of the lower eyelid, situated on a conical elevation called the lacrimal papilla, and surrounded by a fibrous ring [12]. The Riolan muscle and the dense fibrous tissue of the medial eyelid margin insert into the lacrimal punctum and ampulla of the lacrimal drainage system, and together they pull the punctum medially and posteriorly [13]. In order to ensure a proper function of the punctum, these organs should not be severed during punctoplasty. It is not clear what tissue is severed in snip punctoplasty since no tissue is retrieved for biopsy. In Kelly punch punctoplasty, some of the tissue is being removed, allowing us to evaluate it histologically.

Therefore, the purpose of this study was to examine the clinical presentation, histopathological characteristics and surgical success of patients that underwent Kelly punch punctoplasty. To the best of our knowledge, this is the first study that examines histopathological characteristics after Kelly punch punctoplasty.

\section{Methods}

This is a retrospective consecutive case series looking at the clinical and histopathological characteristics of patients that underwent Kelly punch punctoplasty.

The study was approved by the local institutional review board of Sheba Medical Center. All operations were performed by a single surgeon (AP).

\section{Patients}

All patients (without excluding patients due to systemic disease or medications) were diagnosed with punctal stenosis, and referred to Kelly punch punctoplasty and minimonoka placement between January 2017 and December 2017.

The following parameters were retrieved from the medical database and analyzed: patient demographics (gender and age at diagnosis), medical history and medications, main complaint, degree of epiphora (see below), bestcorrected visual acuity (BCVA) before and after the surgery, surgical procedure, follow-up including medical treatment after surgery, post-surgical degree of epiphora, second procedure and restenosis. Snellen VA was converted to $\log$ MAR value.
Moreover, a histopathological examination of the haematoxylin and eosin (H\&E) slides, as well as Masson trichrome staining, were performed on the specimens retrieved from the Kelly punch at the end of the procedure.

\section{Assessment of epiphora}

All patients were asked before the procedure and at least 3 months after the procedure to grade the degree of epiphora on a typical day in each eye using the Munk scoring system: 0 for no epiphora, 1 for epiphora requiring dabbing with a tissue less than twice daily, 2 for epiphora requiring dabbing two to four times daily, 3 for epiphora requiring dabbing five to ten times daily and 4 for epiphora requiring dabbing more than ten times daily [14].

\section{Kelly punch punctoplasty surgery}

Punctoplasty was performed in all cases under local anaesthesia with $2 \%$ Lidocaine, equally combined with Marcaine adrenaline $(1: 100,000)$. The lower punctum site was identified (in all cases in the study it could be identified) and enlarged circumferentially gently by a punctal dilator. A Kelly Descemet's membrane punch with a 1.0-mm-diameter head and a $0.75-\mathrm{mm}$-deep bite was then inserted into the punctum, and a single punch of tissue was removed from the posterior as well as from the lateral wall. Haemostasis was achieved by compression with a cotton tip applicator. A mini-monoka stent was then inserted into the patent punctum. Postoperatively, all patients received Dexamycin drops (Neomycin sulfate and Dexamethasone, Teva Pharmaceutical Industries Ltd, Petach Tikva, Israel) for 2 weeks (three times daily tapered down until cessation at 2 weeks), and were under follow-up 2 weeks after surgery, 1 and 3 months after surgery.

\section{Statistical analysis}

Quantitative variables were described as mean, range and standard deviation. Categorical variables were described as absolute and relative frequencies. A $t$-test analysis was carried out in order to compare the degree of epiphora before and after the procedure. The overall significance level was set to an alpha of 0.05. Statistical analyses were performed using JMP Statistical Discovery Software 7.0 (SAS Institute, Cary, NC, USA).

\section{Results}

The series was composed of 28 patients ( 8 males, 20 females) whose mean age at presentation was 64.25 years $(64.25 \pm 25.2$, range $30-86$ years). 


\section{Clinical characteristics}

The past ocular history of all patients was negative for other lacrimal system or eyelid surgeries, two patients had other eye diseases (glaucoma) and no ectropion was reported on clinical examination. Six patients $(21.42 \%)$ used artificial tears prior to surgery. Regarding systemic medical history, five patients had diabetes mellitus, six patients had hypertension, one patient had pancreas malignancy, one patient was a smoker and no patient had rheumatologic disease. No patient reported any muscle or connective tissue disease or graft-versus-host disease. No patients received ocular chemotherapy in the past or at the time of surgery.

All patients' complaints before surgery were regarding epiphora: 18 bilateral, 5 right-eye epiphora and 5 left-eye epiphora. Ten of them also complained of bilateral ocular irritation. The average Munk degree of epiphora before surgery was 3 and at least 3 months after surgery was 1.04 (matched pairs, $p<0.0001$ ). Mean BCVA before surgery of the operated eye was 0.247 .

\section{Prognosis}

The mean time from surgery to the first follow-up visit after surgery was 25.28 days ( $25.28 \pm 28.42,6-158$ days).

Patients were followed up for a mean time of 6.88 months $(6.88 \pm 5.03,2.53-14.66)$.

At the end of follow-up, two patients $(7.14 \%)$ had restenosis and underwent second punctoplasty. One of them had Riolan muscle in the histopathological exam of the punctum. Eleven patients (39.28\%) still had complaints of low-degree epiphora, and were treated with lubrication or Fluorometholone eye drops.

Mean BCVA after surgery of the operated eye was 0.238 (matched pairs, $p=0.670$ ).

\section{Histopathological results}

Forty-four specimens of punctal tissue taken from the punch punctoplasty were examined.

In H\&E staining, the endocanalicular and marginal punctal epithelium of all specimens showed stratified squamous epithelium with goblet cells (Fig. 1a, b). The submucosal layer revealed connective tissue with no significant inflammation. The Riolan muscle was not visible in the mucosal layer in 40 specimens (Fig. 1a). In four specimens, the muscle was observed (Fig. 1b). A Masson trichrome staining was performed. This staining ensured that the Riolan muscle existed in those four specimens (Fig. 2a, b). Two of the patients with positive masson trichrome staining on histology had good functional outcome and two did not. In seven specimens, chronic inflammation cells were found. No connective tissue for fibrosis were found.

\section{Discussion}

Kelly punch punctoplasty is a simple and less invasive procedure than 1-4-snip punctoplasty. So far, there are only two series summarizing the functional success of this procedure $[15,16]$. However, none of them dealt with the histological appearance.

Our series demonstrates a functional success for Kelly punch punctoplasty, but moreover, it demonstrates for the first time that the muscle of Riolan is not damaged in most cases of Kelly punch punctoplasty.

Punctoplasty using the Kelly punch is performed posteriorly and laterally. Two direction dilation creates a wide enough opening. Punctoplasty is not created anteriorly in order to avoid anterior displacement of the punctum. Also, it is not performed medially to keep the punctum round nasally, in order to decrease the chance for cheese wiring in cases that the patient will need a silicone Crawford stenting in the future $[15,16]$.

The anatomic structure, physiologic performance and pumping mechanism of the lacrimal system are complex and not fully understood. It was hypothesized that Riolan muscle plays a major role in the closure of the lids and the punctum. It probably initiates the sphincter type of closure effected by the orbital portion of orbicularis muscle [17]. Moreover, it probably has a role in the positioning of the punctum and in the pumping mechanism of the tears flowing into the lacrimal sac through the canaliculis [17].

Hur et al. categorized punctal stenosis types based on their macroscopic shape and performed a histopathological analysis for 14 specimens obtained from a punctoplasty procedure (three-snip operation) to assess the characteristics of each stenotic punctal shape. They categorized four shapes: membranous type, slit type, horseshoe type and pinpoint type. They found that Riolan muscle was significantly visible in four specimens $(28.57 \%)$ immediately beneath the mucosal layer, and in four specimens (28.57\%) few muscle fibres were observed, while in six specimens (42.85\%) no significant muscle fibres observed [18]. In our series, in most cases the muscle was not seen and in only four specimens $(4.76 \%)$ muscle fibres were observed. This finding suggests that Kelly punch punctoplasty is a controlled procedure. We assume that Kelly punch punctoplasty creates a custom-sized specimen that does not violate the muscle surrounding the punctum. Riolan muscle in its medial part is $\sim 0.85 \mathrm{~mm}$ [15] and the Kelly punch cut is $<0.75 \mathrm{~mm}$. The circular muscle is not at the orifice of the punctum, it is deeper to the squamous epithelium. Therefore, the muscle will never be completely severed by the Kelly punch-controlled size bite.

The presence of muscle in the specimen is variable among patients. That could be explained either by histologic changes such as hypertrophy of the epithelial antrum of the 
Fig. 1 Haematoxylin and eosin staining of specimen of the punctum. a Slide without a muscle: asterisks-stratified squamous epithelium with goblet cells; arrow-connective tissue with no muscle $(200 \mu \mathrm{m})$. b Slide with a muscle: asterisks —stratified squamous epithelium with goblet cells; arrow-Riolan muscle $(500 \mu \mathrm{m})$.

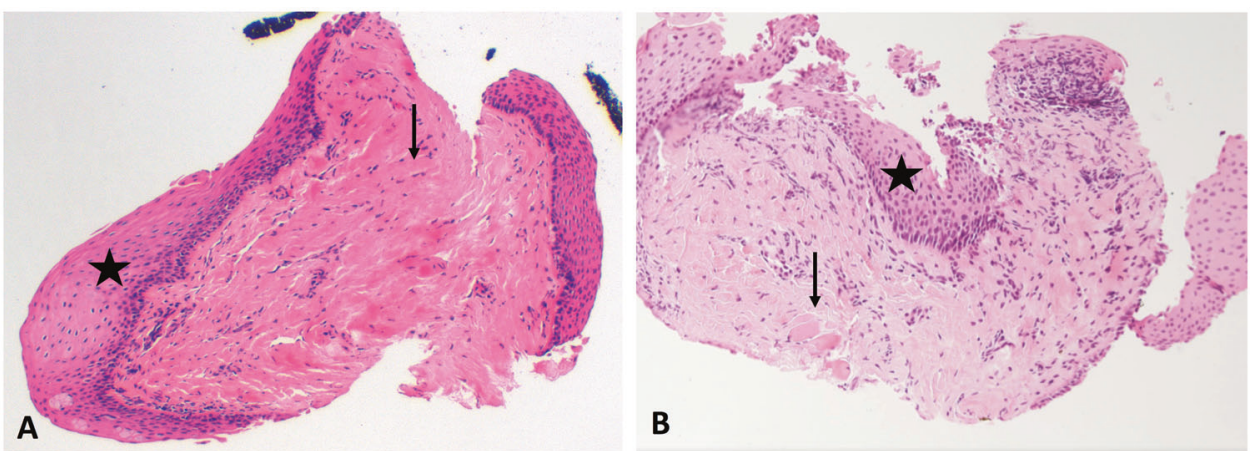

Fig. 2 A Masson trichrome staining of specimen of the punctum. a Slides without a muscle: arrow-connective tissue with no muscle $(200 \mu \mathrm{m})$. b Slide with a muscle: arrowconnective tissue with muscle $(500 \mu \mathrm{m})$.

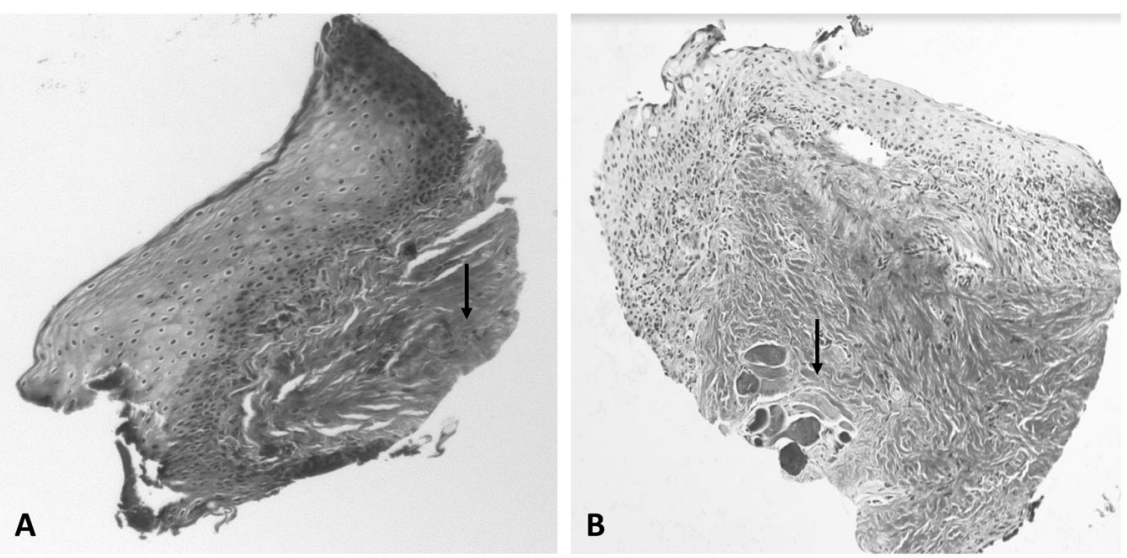

ampulla, fibrotic tissue or atrophic muscle fibres. Moreover, the amount of tissue removed can vary due to the amount of pressure applied by the surgeon on the tissue removed. Very light pressure would excise a smaller than $0.75 \mathrm{~mm}$ of tissue. If a small specimen is removed, the muscle might not appear at all. The important rule is that the Riolan muscle could not be completely severed by using the Kelly punch as mentioned earlier.

In traditional snip punctoplasty, a cut through the edge of the punctum is performed. Those cuts are not uniform and are not controlled. Therefore, the canaliculus itself may be damaged. Moreover, the bleeding and subsequent healing may create fibrosis and therefore restenosis. In Kelly punch punctoplasty, a controlled enlargement of the punctum is done with less bleeding and probably less damage to the canaliculus circular structure [15].

In histopathological specimens, in all but four of the cases using Kelly punch punctoplasty, there is no sign for muscle, while in snip punctoplasty, muscle fibres were found in the specimen in 32.9\% [18]. Intact Riolan muscle enables the punctum to stay in the right circular shape and position, therefore keeping the lacrimal pump intact.

Literature review shows superior success rates with Kelly punch punctoplasty. Wong et al. reported $94 \%$ of anatomical success and $92 \%$ functional success [13]. Vahdani et al. reported about $95 \%$ of anatomical success and $90 \%$ functional success [16]. In our series, $92 \%$ of the patients did not develop restenosis, therefor did not need surgery. This series is the third series to support the superiority of Kelly punch punctoplasty as a primary tool for the treatment of punctual stenosis.

In our institution, before using the Kelly punch, we dilated the puncta by a dilator, and then inserted a minimonoka stent. When shifting to the Kelly method, the rationale was that the stent might be even more important since we are not only dilating an existing opening, but we are dilating by removing tissue, leaving the tissue to heal by scarring. Doing so, we believed that keeping the opening by stenting with mini-monoka would prevent it from adhering in the wrong fashion. However, mini-monoka stenting might not be required.

The drawbacks of this study are its retrospective nature and the absence of a control group. Furthermore, larger, controlled and prospective studies should be done in order to examine this technique. We did not characterize the morphology of the punctum since the specimens removed from the punctum were not the sphincter at whole. Further studies regarding the morphology of the punctum and the role of Riolan muscle in pumping mechanism should be performed. Also, we did not evaluate the procedure with 
and without mini-monoka placement; further studies should evaluate the benefit of using mini-monoka as part of this procedure.

In conclusion, Kelly punch punctoplasty is a simple and minimally invasive procedure with high functional success rate. It is a controlled procedure with a lower chance of damage to the canaliculus and the muscular structure. We believe that Kelly punch punctoplasty with mini-monoka placement is the best surgical procedure to treat isolated punctual stenosis.

\section{Summary}

\section{What was known before}

- Punctal stenosis is a common reason for epiphora-in 2011, Carrim et al. suggested to use Kelly Descemet's membrane punch for punctoplasty, and since then this procedure became more popular.

- It is not clear what tissue is severed in snip punctoplasty since no tissue is retrieved for biopsy.

\section{What this study adds}

- Kelly punch punctoplasty is a simple and minimally invasive procedure with high functional success rate.

- It is a controlled procedure with a lower chance of damage to the canaliculus and the muscular structure.

- We believe that Kelly punch punctoplasty with minimonoka placement is the best surgical procedure to treat isolated punctual stenosis.

\section{Compliance with ethical standards}

Ethical approval All procedures performed in studies involving human participants were in accordance with the ethical standards of the institutional and/or national research committee, and with the 1964 Helsinki declaration and its later amendments or comparable ethical standards.

Informed consent Informed consent was obtained from all individual participants included in the study.

Conflict of interest The authors declare that they have no conflict of interest.
Publisher's note Springer Nature remains neutral with regard to jurisdictional claims in published maps and institutional affiliations.

\section{References}

1. Kashkouli MB, Beigi B, Murthy R, Astbury N. Acquired external punctal stenosis: etiology and associated findings. Am J Ophthalmol. 2003;136:1079-84.

2. Soiberman U, Kakizaki H, Selva D, Leibovitch I. Punctal stenosis: definition, diagnosis, and treatment. Clin Ophthalmol. 2012;6:1011-8.

3. Bowman W. On a new method of treatment applicable to certain cases of epiphora. Med Chir Trans. 1851;34:337-46.

4. Singh S, Ali MJ, Mohamed A. Comparison of outcomes of 3-snip punctoplasty versus simple punctal dilatation with monocanalicular intubation for acquired punctal stenosis. Ophthalmic Plast Reconstr Surg. 2018;34:375-7.

5. Shoaib KK. Outcomes of 4-snip punctoplasty for severe punctal stenosis: measurement of tear meniscus height by optical coherence tomography. Am J Ophthalmol. 2012;154:209. author reply 209-210.

6. Ma'luf RN, Hamush NG, Awwad ST, Noureddin BN. Mitomycin $\mathrm{C}$ as adjunct therapy in correcting punctal stenosis. Ophthalmic Plast Reconstr Surg. 2002;18:285-8.

7. Hussain RN, Kanani H, McMullan T. Use of mini-monoka stents for punctal/canalicular stenosis. Br J Ophthalmol. 2012;96:671-3.

8. Ali MJ, Ayyar A, Naik MN. Outcomes of rectangular 3-snip punctoplasty in acquired punctal stenosis: is there a need to be minimally invasive? Eye. 2015;29:515-8.

9. Hughes WL, Maris CS. A clip procedure for stenosis and eversion of the lacrimal punctum. Trans Am Acad Ophthalmol Otolaryngol. 1967;71:653-5.

10. Edelstein J, Reiss G. The wedge punctoplasty for treatment of punctal stenosis. Ophthalmic Surg. 1992;23:818-21.

11. Carrim ZI, Liolios VI, Vize CJ. Punctoplasty with a kelly punch. Ophthalmic Plast Reconstr Surg. 2011;27:397-8.

12. Jones LT. The cure of epiphora due to canalicular disorders, trauma and surgical failures on the lacrimal passages. Trans Am Acad Ophthalmol Otolaryngol. 1962;66:506-24.

13. Lipham WJ, Tawfik HA, Dutton JJ. A histologic analysis and three-dimensional reconstruction of the muscle of riolan. Ophthalmic Plast Reconstr Surg. 2002;18:93-98.

14. Munk PL, Lin DT, Morris DC. Epiphora: treatment by means of dacryocystoplasty with balloon dilation of the nasolacrimal drainage apparatus. Radiology. 1990;177:687-90.

15. Wong ES, Li EY, Yuen HK. Long-term outcomes of punch punctoplasty with kelly punch and review of literature. Eye. 2017;31:560-5.

16. Vahdani K, Sian I, Giasin O, Makrygiannis G. Functional and anatomical outcomes of punctoplasty with kelly punch. Eye. 2017;31:1628.

17. Mackie IA. Riolan's muscle: action and indications for botulinum toxin injection. Eye. 2000;14:347-52.

18. Hur MC, Jin SW, Roh MS, Jeong WJ, Ryu WY, Kwon YH, et al. Classification of lacrimal punctal stenosis and its related histopathological feature in patients with epiphora. Korean J Ophthalmol. 2017;31:375-82. 